18

\title{
Моделирование спектральных характеристик спонтанной селективной флуоресценции двух различных двухуровневых взаимодействующих наночастиц
}

\author{
(C) В.А. Морозов
}

Институт органической химии им. Н.Д. Зелинского РАН, 119991 Москва, Россия

e-mail: morozov@mail.ioc.ac.ru

Поступила в редакцию 04.08.2021 г.

В окончательной редакции 30.08.2021 г.

Принята к публикации 03.09.2021 г.

\begin{abstract}
Численное моделирование спектральных характеристик спонтанной флуоресценции связанных динамическим взаимодействием двух различных двухуровневых наночастиц при первоначальном возбуждении одной из них выполнено на основе решений уравнений Шредингера для амплитуд несовместимых альтернатив вероятности регистрации состояний составной системы из частиц и квантованного поля излучения при таких поглощениях фотона окружающей средой, которые определяют „пути“ его излучения той или иной частицей. Зависимость интенсивности такой селективной флуоресценции от частоты регистрируемого фотона представлена в виде взаимно перекрывающихся контуров двух пар спектральных линий, отражающих расщепление линий флуоресценции невзаимодействующих частиц. Контуры линий различаются значениями их максимумов. Разности интегральной интенсивности контуров сопоставлены с селективным заселением основного состояния каждой из частиц в ансамбле рассматриваемых пар частиц.
\end{abstract}

Ключевые слова: математическое моделирование, взаимодействующие двухуровневые наночастицы, спонтанная флуоресценция, спектральные характеристики.

DOI: $10.21883 /$ OS.2021.12.51749.2610-21

\section{Введение}

Результаты моделирования динамики спонтанной флуоресценции связанных динамическим взаимодействием двухуровневых наночастиц и соответствующей спектральной характеристики этой флуоресценции лежат в основе интерпретации данных экспериментальных изучений строения и характера взаимодействия возбуждаемых светом двух изолированных атомов, ионов, полупроводниковых квантовых точек, а при рассмотрении таких частиц в качестве физической реализации носителей информации (кубитов) результаты такого моделирования используют для изучения особенностей различных аспектов проявления квантовой перепутанности их состояний как ресурса для развития многих различных приложений в области квантовой информации и для описания различных основных логических операций при квантовых вычислениях (см., например, [1] и приведенную там литературу). Проводимое в многочисленных работах разных авторов отмеченное моделирование основано на использовании формализма оптических уравнений Блоха (ОУБ), которые отражают изменение во времени элементов матрицы плотности составной системы из двух взаимодействующих друг с другом двухуровневых частиц с использованием в качестве базисных их коллективные состояния при полуклассическом описании флуоресценции с феноменологическим учетом экспоненциального затухания воз- бужденного состояния каждой частицы. Однако для полного и детального описания всех характеристик флуоресценции такой пары частиц желательно также иметь информацию относительно динамики состояний каждой из взаимодействующих частиц и соответствующей флуоресценции в отдельности. Такое раздельное (селективное) описание флуоресценции необходимо иметь при составлении представления о механизме фотосенсибилизации различных физических и фотохимических процессов вторичной фотохимической реакции, при описании которой используют такую пару частиц в качестве модели молекулы реагента и молекулы продукта в реакционном центре, как, например, в димере молекул этилена при моделировании каждой из этих молекул двухуровневой частицей [2]. Селективное описание заселенности основного состояния каждой из частиц такой пары позволит определить эффективность рассматриваемого их вторичного фотопревращения и дать полную интерпретацию спектральных характеристик зарегистрированной при этом флуоресценции.

Селективное описание динамики состояний взаимодействующих частиц требует, как отмечено в [3] (стр. 46, 54), использования более общего формализма по сравнению с отмеченным полуклассическим формализмом ОУБ, рассматривающим пару частиц как замкнутую квантовую систему. Таким более общим формализмом для описания динамики состояний спон- 
танной флуоресценции пары частиц является формализм описания состояний полной системы, состоящей из подсистемы, содержащей эти частицы, и подсистемы, содержащей квантованное поле излучения, при рассмотрении этой полной системы как открытой квантовой системы, над которой производятся измерения характеристик ее состояний при непрерывной регистрации (поглощении) фотонов естественным макроскопическим окружением [4-7] или специальным детектором (счетчиком числа фотонов). При этом в квантовой теории измерений принимается во внимание, что регистрация поглощения фотона флуоресценции влияет на состояния излучающей подсистемы, и при описании этого влияния гильбертово пространство состояний поля флуоресценции может рассматриваться как разделенное (стр. 123 в [4], см. также [8]) на 2 подпространства, используемые для моделирования динамики ,индивидуальной траектории“ состояний каждой из отмеченных подсистем в этих подпространствах (описания селективной флуоресценции). Например, при таком моделировании, которое отражает возможность представления о том, какая из взаимодействующих частиц излучила фотон флуоресценции (информация „который путь излучения фотона“ см. гл. 20 в [6]), притом, что сохраняется использование представления о мысленных коллективных состояниях частиц, однако в каждой индивидуальной траектории можно обнаружить отображение (след) реального спектроскопического перехода в одной из взаимодействующих частиц, который - по [9] - заканчивается гейзенберговским квантовым скачком в ее основное состояние при регистрации соответствующего этому переходу фотона флуоресценции.

Заметим, что в области квантовой информации такое представление о селективной заселенности основного состояния каждой из пары частиц при флуоресценции связывают с квантовым преобразованием [10] и трактуют как „мгновенное открытие замкнутой системы из частиц“ при измерении определенных характеристик состояний поля излучения и дискретных характеристик состояний частиц (см. стр. 441 в [10]) с „отбрасыванием“ (как отмечено на стр.46 в [3]) всех остальных состояний.

Результаты применения такого подхода к использованию отмеченного общего формализма при моделировании спектральных характеристик спонтанной селективной флуоресценции двух различных двухуровневых наночастиц описаны в настоящей статье. Во второй части статьи приведено описание модели рассматриваемой полной составной системы и решение уравнений, в третьей части - анализ полученных выражений для контуров спектральных линий флуоресценции и в четвертой части - соответствующие иллюстрации примеров вида и расположения этих контуров. Статья заканчивается заключительным замечанием.

\section{Модель системы и решение уравнений}

Гамильтониан полной составной системы из близко расположенных друг относительно друга двух точечных, неподвижных двухуровневых частиц (частица $A$ и частица $B)$, которые взаимодействуют между собой и с квантованным полем излучения, запишем в виде:

$$
H=W+\sum_{j=A, B}{ }_{j} H+{ }_{j} V+H_{f},
$$

где ${ }_{A} H\left({ }_{B} H\right)$ - гамильтониан частицы $A(B) ;{ }_{A} V\left({ }_{B} V\right)-$ оператор энергии взаимодействия частицы $A$ (частицы $B)$ с поперечными компонентами поля излучения в электродипольном приближении; ${ }_{A} V=-\left(\mathbf{e d}_{A}\right)$, где $\mathbf{e}(t)$ - напряженность электрического поля в точке расположения частицы $A ; \mathbf{d}_{A}-$ оператор дипольного момента частицы $A ;{ }_{B} V=-\left(\mathbf{e d}_{B}\right)$, где $\mathbf{e}(t)-$ напряженность электрического поля в точке расположения частицы $B, \mathbf{d}_{B}-$ оператор дипольного момента второй частицы $B$; расстояние между точками расположения частиц много меньше длины волны собственной спонтанной флуоресценции свободной частицы $A$ и собственной спонтанной флуоресценции свободной частицы $B$; $H_{f}$ - гамильтониан поля излучения. Под оператором $W$ будем подразумевать оператор энергии кулоновского диполь-дипольного взаимодействия распределения зарядов частиц, приводящего к обратимому обмену энергией возбуждения между частицами в таких коллективных состояниях, когда одна частица находится в возбужденном состоянии, а другая - в основном (см. ниже).

На рис. 1 схематически приведено относительное положение отмеченных отрезками горизонтальных линий уровней энергии $E_{n}$ собственных состояний $|n\rangle$ $(n=1,4)$ частицы $A$ и $|n\rangle(n=2,3)$ частицы $B$. Для простоты рассмотрения приведены примерно равные значения разности $E_{4}-E_{3}>0$ и $E_{2}-E_{1}>0$ (как обычно принимается при рассмотрении моделирования динамики состояний взаимодействующих ионов атомов см. рис. 1 в [11]), но можно полагать, что $E_{2}=E_{1}$, как во

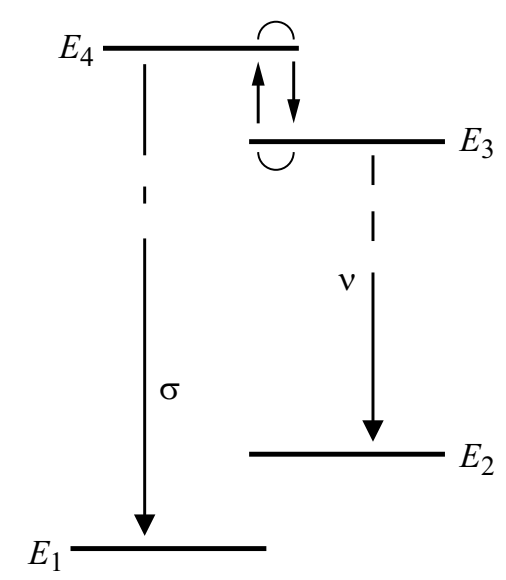

Рис. 1. Схема относительного положения уровней энергии частиц с указанием переходов между ними. 
многих работах по изучению динамики перепутанности состояний двух двухуровневых частиц - см., например, [12] и приведенную там литературу.

Тонкими линиями со стрелками обозначены радиационные переходы при излучении отмеченных выше фотонов собственной спонтанной флуоресценции изолированных частиц, а круговыми кривыми - нерадиационные обратимые переходы между такими коллективными состояниями, когда одна частица находится в возбужденном состоянии, а другая — в основном. Эти переходы обусловлены кулоновским диполь-дипольным взаимодействием, как, например, взаимодействие близко отстоящих друг от друга двух щелочно-земельных атомов, соответствие геометрии расположения которых и биение интенсивности их флуоресценции изучалось в [13], или взаимодействие пары квантовых точек $\mathrm{CdSe} / \mathrm{ZnS}$, геометрия относительного положения которых определялась по мерцанию их флуоресценции в [14].

Заметим, что в ряде работ при моделировании динамики перепутанности состояний в системе двух динамически („когерентно“) взаимодействующих двухуровневых частиц принималось (см., например, [15]), что каждая из частиц независимо взаимодействует со своим отдельным радиационным резервуаром.

Учитывая, что невзаимодействующие наночастицы часто характеризуются тем, что полосы, образуемые спектральными линиями их собственного излучения, практически не перекрываются, будем считать, что частицы излучают в общий радиационный резервуар, и ограничимся рассмотрением состояний поля флуоресценции, которые содержат один фотон. Обозначим „сорт“ этого фотона (совокупность индексов, характеризующих его волновой вектор и состояние поляризации) буквой $\mu$, и среди состояний поля $|\mu\rangle$ в гильбертовом пространстве фотонов флуоресценции будем различать состояния сорта $|\sigma\rangle$ и состояния сорта $|v\rangle(\sigma, v \in \mu)$, которые соответственно относятся к состояниям фотона спонтанного излучения при переходе $|4\rangle \rightarrow|1\rangle$ частицы $A-$ как изолированной, так и взаимодействующей с частицей $B-$ и при переходе $|3\rangle \rightarrow|2\rangle$ частицы $B-$ как изолированной, так и взаимодействующей с частицей $A$. Начальное состояние поля без фотонов будем обозначать как $|0\rangle$.

Для объема $L^{3}$ пространства, в котором находятся частицы и рассматриваемое однофотонное поле излучения, при введенных обозначениях имеем для матричного элемента оператора ${ }_{A} V$ по состояниям полной составной системы $|12 \mu\rangle$ и $|420\rangle$ такое выражение: ${ }_{A} V_{1 \mu}^{40}=i L^{-3 / 2} \sqrt{2 \pi \hbar \omega_{\mu}}\left(d_{\mu}\right)_{14}$, где $\left(d_{\mu}\right)_{14}-$ матричный элемент проекции оператора $d$ по состояниям $|1\rangle$ и $|4\rangle$ на направление поляризации фотона $\mu$, $\omega_{\mu}$ - частота фотона $\mu$; для матричного элемента оператора ${ }_{B} V$ по состояниям $|12 \mu\rangle$ и $|130\rangle$ - такое выражение: ${ }_{B} V_{2 \mu}^{30}=i L^{-3 / 2} \sqrt{2 \pi \hbar \omega_{\mu}}\left(d_{\mu}\right)_{23}$; где $\left(d_{\mu}\right)_{23}-$ проекция матричного элемента оператора $d$ по состояниям $|2\rangle$ и $|3\rangle$ на направление поляризации фотона $\mu$. Аналогично имеем ${ }_{A} V_{1 \sigma}^{40}=i L^{-3 / 2} \sqrt{2 \pi \hbar \omega_{\sigma}}\left(d_{\sigma}\right)_{14}$ и
${ }_{B} V_{2 v}^{30}=i L^{-3 / 2} \sqrt{2 \pi \hbar \omega_{v}}\left(d_{v}\right)_{23}$. Использование таких матричных элементов оператора взаимодействия частиц и поля излучения соответствует приближенному описанию динамики состояний полной системы, которое учитывает только переходы с сохранением ее энергии, пренебрегая малым вкладом в амплитуду этих состояний от учета виртуальных переходов, при которых энергия полной системы не сохраняется. Такое приближение часто называют „резонансным“ (или приближением вращающейся волны [6]).

Константы $\gamma_{4}, \gamma_{3}$, которые характеризуют скорости радиационного распада состояния $|4\rangle$ изолированной частицы $A$ при излучении фотона $\sigma$ и соответственно состояния $|3\rangle$ изолированной частицы $B$ при излучении фотона $v$ (см., например, [16]), имеют вид:

$$
\begin{aligned}
& \gamma_{4}=\left.\left.\pi \hbar^{-1} \sum_{\sigma}\right|_{A} V_{1 \sigma}^{40}\right|^{2} \delta\left(E_{40}-E_{1 \sigma}\right)=2 \omega_{41}^{3} d_{41}^{2} / 3 \hbar c^{3}, \\
& \gamma_{3}=\left.\left.\pi \hbar^{-1} \sum_{v}\right|_{B} V_{2 v}^{30}\right|^{2} \delta\left(E_{30}-E_{2 v}\right)=2 \omega_{32}^{3} d_{32}^{2} / 3 \hbar c^{3},
\end{aligned}
$$

где $\delta(E)$ - функция Дирака; $\omega_{41}=\hbar^{-1}\left(E_{4}-E_{1}\right)$, $\omega_{32}=\hbar^{-1}\left(E_{3}-E_{2}\right)$.

Предполагается, что $\gamma_{4} \ll \omega_{41}$ и $\gamma_{3} \ll \omega_{32}$, так что, как это уже отмечено выше, можно пренебрегать перекрыванием контуров спектральных линий собственной флуоресценции изолированных частиц. Эти контуры имеют лоренцеву форму с максимумами при $\omega_{41}$ и $\omega_{32}$, и полушириной $\gamma_{4}$ и $\gamma_{3}$.

Матричные элементы оператора $W$ для нерадиационных переходов между состояниями $|420\rangle$ и $|130\rangle$ будут обозначаться как $\langle 42|W| 13\rangle=\langle 13|W| 42\rangle \equiv \hbar w$.

Моделирование динамики заселенности состояний полной системы основано на использовании решений уравнения Шредингера для амплитуд $b_{k}(t)$ заселенности собственных состояний $k$ этой системы:

$$
i \hbar \dot{b}_{k}(t)=\sum_{l}(V+W)_{k l} b_{l}(t) \exp \left[i\left(E_{k}-E_{l}\right) t / \hbar\right]+i \hbar \delta_{k i} \delta(t),
$$

где $i, k, l$ - индексы базисных ортонормированных состояний, энергия которых соответственно $E_{i}, E_{k}, E_{l} ; i-$ индекс начального состояния; $\delta_{k i}-$ символ Кронекера, $\delta_{k i}=0$ при $k \neq i$ и $\delta_{k i}=1$ при $k=i ; \delta(t)-$ функция Дирака. Время $t>0$ будем отсчитывать от момента $t=0$. Относительно роли неоднородного члена $i \hbar \delta_{k i} \delta(t)$ и сохранения нормировки $\sum_{k}\left|b_{k}(t)\right|^{2}-1-$ см. например гл. 4 в [16].

Система уравнений для амплитуд $b_{k}(t)$, $k \div 420,130,12 \mu$ рассматриваемой пары частиц и фотона флуоресценции $|\mu\rangle$ при начальном условии $b_{420}(+0)=1$ в резонансном приближении имеет вид:

$$
\begin{aligned}
i \hbar \dot{b}_{420}(t)= & \sum_{\mu}{ }_{A} V_{40}^{1 \mu} b_{12 \mu}(t) \exp \left[i\left(E_{420}-E_{12 \mu}\right) t / \hbar\right] \\
& +W_{42}^{13} b_{130}(t) \exp \left[i\left(E_{420}-E_{130}\right) t / \hbar\right]+i \hbar \delta(t),
\end{aligned}
$$




$$
\begin{aligned}
i \hbar \dot{b}_{130}(t)= & \sum_{\mu}{ }_{B} V_{30}^{2 \mu} b_{12 \mu}(t) \exp \left[i\left(E_{130}-E_{12 \mu}\right) t / \hbar\right] \\
& +W_{13}^{42} b_{420}(t) \exp \left[i\left(E_{130}-E_{420}\right) t / \hbar\right], \\
i \hbar \dot{b}_{12 \mu}(t)= & { }_{A} V_{1 \mu}^{40} b_{420}(t) \exp \left[i\left(E_{12 \mu}-E_{420}\right) t / \hbar\right] \\
& +{ }_{B} V_{2 \mu}^{30} b_{130}(t) \exp \left[i\left(E_{12 \mu}-E_{130}\right) t / \hbar\right] .
\end{aligned}
$$

Относительно создания такого начального условия см., например, в [13].

Рассмотрим решение этой системы уравнений при использовании фурье-представления

$$
b_{k}(t)=i(2 \pi)^{-1} \int_{-\infty}^{+\infty} G_{k}(E) \exp \left[i\left(E_{k}-E\right) t / \hbar\right] d E
$$

где $E-$ энергетическая переменная и

$$
\left(E-E_{k}\right) G_{k}(E)=\sum_{l}(V+W)_{k l} G_{l}(E)+\delta_{k i} .
$$

Используем обозначения:

$$
\begin{gathered}
i \hbar \gamma_{4}=-\sum_{\sigma}\left|{ }_{A} V_{1 \sigma}^{40}\right|^{2} \xi\left(E-E_{12 \sigma}\right) ; \\
i \hbar \gamma_{3}=-\sum_{\nu}\left|{ }_{B} V_{2 \nu}^{30}\right|^{2} \xi\left(E-E_{12 v}\right), \\
i \hbar \gamma_{(43)}=-\sum_{\mu}{ }_{A} V_{40}^{1 \mu}{ }_{2} V_{2 \mu}^{30} \xi\left(E-E_{12 \mu}\right)=i \hbar \gamma_{(34)},
\end{gathered}
$$

где $\xi(E)=P / E-i \pi \delta(E), P / E-$ главное значение функции $1 / E$, и как обычно (см., например, [16]) под выражениями $\gamma_{m}(m \div 4,3)$ будем подразумевать приведенные выше константы $\gamma_{4}$ и $\gamma_{3}$, а под выражениями $\gamma_{(43)}$ и $\gamma_{(34)}-$ результат суммирования по сортам фотонов $\mu$ приведенных выше выражений при замене в них $\zeta\left(E-E_{12 \mu}\right)$ на $-i \pi \delta\left(E-E_{12 \mu}\right)$.

Заметим, что динамику заселенности возбужденных состояний рассматриваемых двухуровневых частиц можно описывать подобным же образом и в том случае, когда они взаимодействуют и с полем квантованных осцилляторов окружающего их теплового резервуара (фононов) (см. приложение 1А в [6] и гл. 8 в [17]). При этом можно использовать уравнения и выражения, описывающие появление фононов в тепловом резервуаре, аналогично приведенным выше уравнениям и выражениям, полученным при описании излучения фотона в радиационный резервуар. Имея это в виду, в качестве дополнительного - к радиационным переходам - учета распадов возбужденных состояний частиц с переходом в основное состояние с рождением фононов окружения будем полагать, что константы $\gamma_{3}$ и $\gamma_{4}$ учитывают и радиационный и нерадиационный распады возбужденных состояний молекул, как это часто принято в формализме ОУБ, в котором соответствующие константы называют константами скорости продольной релаксации (приложение 5.5 в $[6])$.
Используя введенные обозначения, решаем систему уравнений для $G_{n}(E)$ при $b_{420}(t=0)=1$ и получаем следующие выражения:

$$
\begin{aligned}
& G_{420}(E)= \\
& \frac{E-E_{130}+i \hbar \gamma_{3}}{\left(E-E_{420}+i \hbar \gamma_{4}\right)\left(E-E_{130}+i \hbar \gamma_{3}\right)-\hbar^{2}\left(w-i \gamma_{(43)}\right)^{2}}, \\
& G_{130}(E)=\frac{\hbar\left(w-i \gamma_{(43)}\right)}{E-E_{130}+i \hbar \gamma_{3}} G_{420}(E), \\
& G_{12 \mu}(E)= \\
& \frac{{ }_{A} V_{12 \mu}^{420}\left(E-E_{130}+i \hbar \gamma_{3}\right)+{ }_{B} V_{12 \mu}^{130} \hbar\left(w-i \gamma_{(43)}\right)}{\left(E-E_{420}+i \hbar \gamma_{4}\right)\left(E-E_{130}+i \hbar \gamma_{3}\right)-\hbar^{2}\left(w-i \gamma_{(43)}\right)^{2}} \\
& \times \xi\left(E-E_{12 \mu}\right) . \\
& a^{2} \equiv w^{2}-\tilde{\gamma}^{2}, \\
& E_{420}-E_{130}=\hbar\left(\omega_{41}-\omega_{32}\right)=\hbar\left(\omega_{43}+\omega_{21}\right) \equiv 2 \hbar(\Delta+\bar{\Delta}) \equiv 2 \hbar \tilde{\Delta} .
\end{aligned}
$$

С использованием этих обозначений нули знаменателей в приведенных выражениях $G_{n}\left(E \equiv \hbar x+E_{130}\right)$ определяются решениями уравнения

$$
x^{2}+2(i \bar{\gamma}-\tilde{\Delta}) x-2 i \tilde{\Delta} \gamma_{3}-\gamma_{3} \gamma_{4}-\left(w-i \gamma_{(43)}\right)^{2}=0 .
$$

Корни этого уравнения имеют вид:

$$
\begin{gathered}
x_{1,2}=\tilde{\Delta}-i \bar{\gamma} \pm(\alpha-i \beta)=\tilde{\Delta} \pm \alpha-i(\bar{\gamma} \pm \beta), \\
\alpha=(\sqrt{2})^{-1} \sqrt{\sqrt{A^{2}+B^{2}}+A}, \\
\beta=-(\sqrt{2})^{-1} \sqrt{\sqrt{A^{2}+B^{2}}-A},
\end{gathered}
$$

где $A=\tilde{\Delta}^{2}+w^{2}+\gamma_{3} \gamma_{4}-\bar{\gamma}^{2}-\gamma_{(43)}^{2}, B=-2\left(w \gamma_{(43)}-\tilde{\gamma} \tilde{\Delta}\right)$.

Знаменатели выражений $G_{n}(E)$ запишем в виде $\hbar^{2}\left(x-x_{1}\right)\left(x-x_{2}\right)$, так что корни $x_{1,2}$ представляют собой полюсы в нижней части аналитического продолжения этих функций в нижнюю комплексную полуплоскость.

Используя полученные выражения для $G_{n}(E)$, теорию вычетов для этих функций, и соотношения

$$
\begin{gathered}
\lim _{t \rightarrow \infty} \xi\left(E-E_{1 \sigma}\right) \exp \left[i\left(E_{1 \sigma}-E\right) t / \hbar\right]=-2 \pi i \delta\left(E-E_{1 \sigma}\right), \\
\int_{-\infty}^{+\infty} f(E) \delta\left(E-E_{1 \sigma}\right) d E=f\left(E_{1 \sigma}\right),
\end{gathered}
$$

для $t \gg 1 / \gamma_{4}$ получаем:

$$
\begin{aligned}
& b_{12 \mu}\left(\bar{\omega}_{\mu}, t=\infty\right)=i(2 \pi)^{-1} \lim _{t \rightarrow \infty} \int_{-\infty}^{+\infty} G_{12 \mu}(E) \\
& \quad \times \exp \left[i\left(E_{12 \mu}-E\right) t / \hbar\right] d E=\left[{ }_{A} V_{1 \mu}^{40}\left(\bar{\omega}_{\mu}+2 \tilde{\Delta}+i \gamma_{3}\right)\right. \\
& \left.\quad+{ }_{B} V_{2 \mu}^{30}\left(w-i \gamma_{(34)}\right)\right] / F_{\mu}\left(\bar{\omega}_{\mu}\right),
\end{aligned}
$$


где $\quad \bar{\omega}_{\mu} \equiv \omega_{\mu}-\omega_{41} \quad$ и $\quad F_{\mu}\left(\bar{\omega}_{\mu}\right)=\hbar\left(\bar{\omega}_{\mu}+\tilde{\Delta}-\alpha-i \bar{\gamma}-i \beta\right)$ $\times\left(\bar{\omega}_{\mu}+\tilde{\Delta}+\alpha-i \bar{\gamma}+i \beta\right)$.

Амплитуда $b_{12 \mu}\left(\bar{\omega}_{\mu}, t=\infty\right)$ имеет вид суммы слагаемых, одно из которых пропорционально ${ }_{A} V_{1 \mu}^{40}$, а другое $-{ }_{B} V_{2 \mu}^{30}$, так что эта амплитуда отображает интерферирующие „альтернативы“ (см. стр. 25 в [18]) вероятности излучения фотона по каналу излучения $|4\rangle \rightarrow|1\rangle$ и по каналу излучения $|3\rangle \rightarrow|2\rangle$, или, можно сказать, излучения частицей $A$, взаимодействующей с частицей $B$, и излучения частицей $B$, взаимодействующей с частицей $A$.

Примем теперь во внимание отмеченную во Введении возможность описания выбора того или иного „пути излучения фотона“ при его регистрации макроскопическим окружением. Для моделирования процесса соответствующей селективной флуоресценции используется разделение гильбертова пространства состояний поля флуоресценции рассматриваемой полной системы $|12 \mu\rangle$ на два подпространства состояний: $|12 \sigma\rangle$ и $|12 v\rangle$. Состояние $|12 \sigma\rangle$ относится к полю излучения частицей $A$, взаимодействующей с частицей $B$ (канал $A$ ), а состояние $|12 v\rangle$ - к полю излучения частицей $B$ взаимодействующей с частицей $A$ (канал $B$ ). При этом амплитуды $b_{12 \sigma}$ и $b_{12 v}$ этих состояний отображают несовместимые альтернативы вероятности излучения соответствующего фотона, которые - см. стр. 26 в [18] - могут быть идентифицированы по отдельности.

Описание такого вмешательства в процесс рассматриваемой флуоресценции, которое определяет выбор пути излучения ее фотона - по каналу $A$ или по каналу $B-$ предлагается рассмотреть на основе решения системы уравнений для амплитуд состояний полной системы, которая отличается от приведенной выше системы уравнений Шредингера тем, что помимо уравнений для $i \hbar \dot{b}_{420}(t)$ и $i \hbar \dot{b}_{130}(t)$ содержит два уравнения: одно для $i \hbar \dot{b}_{12 \mu}(t)$ и другое для $i \hbar \dot{b}_{12 v}(t)$, тогда как приведенная система уравнений Шредингера помимо таких же уравнений для $i \hbar \dot{b}_{420}(t)$ и $i \hbar \dot{b}_{130}(t)$ содержит одно уравнение для $i \hbar \dot{b}_{12 \mu}(t)$.

Предлагаемая система уравнений выглядит следующим образом:

$$
\begin{aligned}
i \hbar \dot{b}_{420}(t)= & \sum_{\sigma}{ }_{A} V_{40}^{1 \sigma} b_{12 \sigma}(t) \exp \left[i\left(E_{420}-E_{12 \sigma}\right) t / \hbar\right] \\
& +W_{42}^{13} b_{130}(t) \exp \left[i\left(E_{420}-E_{130}\right) t / \hbar\right]+i \hbar \delta(t), \\
i \hbar \dot{b}_{130}(t)= & \sum_{v}{ }_{B} V_{30}^{2 v} b_{12 v}(t) \exp \left[i\left(E_{130}-E_{12 v}\right) t / \hbar\right] \\
& +W_{13}^{42} b_{420}(t) \exp \left[i\left(E_{130}-E_{420}\right) t / \hbar\right], \\
i \hbar \dot{b}_{12 \sigma}(t)= & { }_{A} V_{1 \sigma}^{40} b_{420}(t) \exp \left[i\left(E_{12 \sigma}-E_{420}\right) t / \hbar\right], \\
i \hbar \dot{b}_{12 v}(t)= & { }_{B} V_{2 v}^{30} b_{130}(t) \exp \left[i\left(E_{12 v}-E_{130}\right) t / \hbar\right] .
\end{aligned}
$$

Используя соответствующую систему уравнений для $G_{n}(E)$ при $b_{420}(t=0)=1$, получаем следующие выражения:

$$
\begin{aligned}
G_{420}(E)= & \frac{E-E_{130}+i \hbar \gamma_{3}}{\left(E-E_{420}+i \hbar \gamma_{4}\right)\left(E-E_{130}+i \hbar \gamma_{3}\right)-(\hbar w)^{2}}, \\
& G_{130}(E)=\frac{\hbar w}{E-E_{130}+i \hbar \gamma_{3}} G_{420}(E), \\
& G_{12 v}(E)={ }_{B} V_{2 v}^{30} \xi\left(E-E_{12 v}\right) G_{130}(E), \\
& G_{12 \sigma}(E)={ }_{A} V_{1 \sigma}^{40} \xi\left(E-E_{12 \sigma}\right) G_{420}(E) .
\end{aligned}
$$

C использованием приведенных выше обозначений нули знаменателей в приведенных выражениях $G_{n}\left(E \equiv \hbar x+E_{130}\right)$ определяются решениями уравнения

$$
x^{2}+2(i \bar{\gamma}-\tilde{\Delta}) x-2 i \tilde{\Delta} \gamma_{3}-\gamma_{3} \gamma_{4}-w^{2}=0 .
$$

Корни этого уравнения имеют следующий вид:

$$
x_{1,2}=\tilde{\Delta}-i \bar{\gamma} \pm(\delta-i \eta)=\tilde{\Delta} \pm \delta-i(\bar{\gamma} \pm \eta),|\eta|<\bar{\gamma},
$$

где

$\delta=(\sqrt{2})^{-1} \sqrt{\sqrt{\left(w^{2}-\tilde{\gamma}^{2}+\tilde{\Delta}^{2}\right)^{2}+4 \tilde{\Delta}^{2} \tilde{\gamma}^{2}}+w^{2}-\tilde{\gamma}^{2}+\tilde{\Delta}^{2}}$,

$\eta=-(\sqrt{2})^{-1} \sqrt{\sqrt{\left(w^{2}-\tilde{\gamma}^{2}+\tilde{\Delta}^{2}\right)^{2}+4 \tilde{\Delta}^{2} \tilde{\gamma}^{2}}-w^{2}+\tilde{\gamma}^{2}-\tilde{\Delta}^{2}}$.

Заметим, что при $w=0$ имеем $\delta=\tilde{\Delta}$ и $\eta=-\tilde{\gamma}$, а при $w>0$ имеем $\delta>\tilde{\Delta}$.

Используя соотношения:

$$
\begin{gathered}
\lim _{t \rightarrow \infty} \zeta\left(E-E_{12 \sigma}\right) \exp \left[i\left(E_{12 \sigma}-E\right) t / \hbar\right]=-2 \pi i \delta\left(E-E_{12 \sigma}\right), \\
\int_{-\infty}^{+\infty} f(E) \delta\left(E-E_{12 \sigma}\right) d E=f\left(E_{12 \sigma}\right),
\end{gathered}
$$

и аналогичные соотношения для фотонов v, получаем:

$$
\begin{aligned}
& b_{12 \sigma}\left(\bar{\omega}_{\sigma}, t=\infty\right)=i(2 \pi)^{-1} V_{1 \sigma}^{40} \lim _{t \rightarrow \infty} \int_{-\infty}^{+\infty} \zeta\left(E-E_{12 \sigma}\right) G_{420}(E) \\
& \times \exp \left[i\left(E_{12 \sigma}-E\right) t / \hbar\right] d E=V_{\sigma 1}^{04}\left(\bar{\omega}_{\sigma}+2 \tilde{\Delta}+i \gamma_{3}\right) / F_{\sigma}\left(\bar{\omega}_{\sigma}\right),
\end{aligned}
$$

где $\bar{\omega}_{\sigma} \equiv \omega_{\sigma}-\omega_{41}$ и $F_{\sigma}\left(\bar{\omega}_{\sigma}\right)=\hbar\left(\bar{\omega}_{\sigma}+\tilde{\Delta}-\delta+i \bar{\gamma}+i \eta\right)$ $\times\left(\bar{\omega}_{\sigma}+\tilde{\Delta}+\delta+i \bar{\gamma}-i \eta\right)$.

$$
\begin{aligned}
& b_{12 v}\left(\bar{\omega}_{v}, t=\infty\right)=i(2 \pi)^{-1} V_{2 v}^{30} \lim _{t \rightarrow \infty} \int_{-\infty}^{+\infty} \zeta\left(E-E_{12 v}\right) G_{130}(E) \\
& \times \exp \left[i\left(E_{12 v}-E\right) t / \hbar\right] d E=w V_{2 v}^{30} / F_{v}\left(\tilde{\omega}_{v}\right),
\end{aligned}
$$

где $\tilde{\omega}_{v} \equiv \omega_{v}-\omega_{32}$ и $F_{\nu}\left(\tilde{\omega}_{V}\right)=\hbar\left(\tilde{\omega}_{V}-\tilde{\Delta}-\delta+i \bar{\gamma}+i \eta\right)$ $\times\left(\tilde{\omega}_{V}-\tilde{\Delta}+\delta+i \bar{\gamma}-i \eta\right)$.

Учитывая равенство $\omega_{32}=\omega_{41}-2 \tilde{\Delta}$, перепишем $\tilde{\omega}_{v}$ в виде $\tilde{\omega}_{v} \equiv \omega_{v}-\omega_{32}=\omega_{v}-\omega_{41}+2 \tilde{\Delta} \equiv \bar{\omega}_{v}+2 \tilde{\Delta}$. Используя определение $\bar{\omega}_{v} \equiv \omega_{v}-\omega_{41}$, получаем выражение $F_{v}\left(\bar{\omega}_{\nu}\right)$ в таком же виде, как и выражение $F_{\sigma}\left(\bar{\omega}_{\sigma}\right)$ при замене $\bar{\omega}_{\sigma} \equiv \omega_{\sigma}-\omega_{41}$ на $\bar{\omega}_{v} \equiv \omega_{v}-\omega_{31}$, т. е. имеем $F_{\sigma}\left(\bar{\omega}_{\sigma}\right)=F_{v}\left(\tilde{\omega}_{v}\right)=F_{v}\left(\bar{\omega}_{v}\right) \equiv F(\omega)$ при $\bar{\omega}_{\sigma}=\bar{\omega}_{v} \equiv \omega$. 


\section{Анализ спектров}

Используя квадраты модулей амплитуд $b_{12 \mu}\left(\bar{\omega}_{\mu}, t=\infty\right), \quad b_{12 \sigma}\left(\bar{\omega}_{\sigma}, t=\infty\right), \quad b_{12 v}\left(\bar{\omega}_{\nu}, t=\infty\right)$, можно записать выражения для спектров интенсивности излучения в направлении волнового вектора соответствующего фотона. Например, для фотона v имеем:

$$
J\left(\omega_{v}\right)=\hbar \omega_{v} \rho_{\omega_{v}}\left|b_{2 v}\left(\omega_{v}, \infty\right)\right|^{2},
$$

где $\rho_{\omega_{v}}=\omega_{v}^{2}(2 \pi c)^{-3}-$ плотность состояний поля фотонов $v$ на единицу объема (см., например, стр. 57 в [16]).

При рассмотрении некоторых спектральных характеристик изучаемой флуоресценции можно ограничиться использованием самих безразмерных функций:

$$
\begin{aligned}
\left|b_{12 \mu}\left(\omega_{\mu}, t=\infty\right)\right|^{2} & \equiv S_{12 \mu}\left(\bar{\omega}_{\mu}\right), \\
\left|b_{12 \sigma}\left(\omega_{\sigma}, t=\infty\right)\right|^{2} & \equiv S_{12 \sigma}\left(\bar{\omega}_{\sigma}\right), \\
\left|b_{12 v}\left(\omega_{v}, t=\infty\right)\right|^{2} & \equiv S_{12 v}\left(\bar{\omega}_{v}\right),
\end{aligned}
$$

которые и будем называть соответственно спектром рассматриваемой неселективной $\mu$-флуоресценции, спектром $\sigma$-компоненты селективной флуоресценции и спектром $v$-компоненты селективной флуоресценции или просто, когда это не вызывает недоумения, спектром $\sigma$ флуоресценции и спектром $\mathcal{v}$-флуоресценции.

Спектр $\mu$-флуоресценции имеет следующий вид:

$$
\begin{gathered}
S_{12 \mu}\left(\bar{\omega}_{\mu}\right)=\left\{\left|{ }_{A} V_{1 \mu}^{40}\right|^{2}\left[\left(\bar{\omega}_{\mu}+2 \tilde{\Delta}\right)^{2}+\gamma_{3}^{2}\right]+\left.\left.\right|_{B} V_{2 \mu}^{30}\right|^{2}\left(w^{2}+\gamma_{(34)}^{2}\right)\right. \\
\left.+\left.2\right|_{A} V_{1 \mu}^{40} \|_{B} V_{2 \mu}^{30} \mid\left[w\left(\omega_{\mu}+2 \tilde{\Delta}\right)-\gamma_{3} \gamma_{(34)}\right]\right\} /\left|F_{\mu}\left(\bar{\omega}_{\mu}\right)\right|^{2},
\end{gathered}
$$

где

$$
\begin{aligned}
\left.F_{\mu}\left(\bar{\omega}_{\mu}\right)\right|^{2}= & \hbar^{2}\left[\left(\bar{\omega}_{\mu}+\tilde{\Delta}-\alpha\right)^{2}+(\bar{\gamma}+\beta)^{2}\right] \\
& \times\left[\left(\bar{\omega}_{\mu}+\tilde{\Delta}+\alpha\right)^{2}+(\bar{\gamma}-\beta)^{2}\right] .
\end{aligned}
$$

Этот спектр состоит из двух компонент, которые образуются при расщеплении линии $\omega_{41}$ спонтанной собственной флуоресценции изолированной частицы $A$ под влиянием ее взаимодействия с частицей $B$. Форма, положение, значения максимумов и ширины контуров этих компонент определяются значениями частоты $\omega_{41}$, частоты $\omega_{32}$, константами $\gamma_{3}, \gamma_{4}, w$, расстояния между уровнями энергии $\tilde{\Delta}$ и значениями матричных элементов ${ }_{A} V_{1 \sigma}^{40},{ }_{B} V_{2 v}^{30}$. Так, максимумы этих контуров приходятся на значения частоты $\bar{\omega}_{\mu}=\alpha-\tilde{\Delta}$ и значения частоты $\bar{\omega}_{\mu}=-\alpha-\tilde{\Delta}$, а расстояние между этими максимумами $\cong 2 \alpha>2 \tilde{\Delta}$. Обозначим эти контуры как $S_{A I \mu}\left(\bar{\omega}_{\mu}\right)$ и $S_{A I I \mu}\left(\bar{\omega}_{\mu}\right)$ соответственно. Контур $S_{A I \mu}\left(\bar{\omega}_{\mu}\right)$ сдвинут относительно контура линии собственной флуоресценции $S_{A}\left(\bar{\omega}_{\sigma}\right)$ изолированной частицы $A$ в сторону большей частоты, а контур $S_{A I I \mu}\left(\bar{\omega}_{\mu}\right)-$ в сторону меньшей частоты настолько, что его максимум располагается при меньшем значении частоты максимума контура $S_{B}\left(\bar{\omega}_{\nu}\right)$ собственной флуоресценции частицы $B$ (рис. 2). Числитель выражения $S_{12 \mu}\left(\bar{\omega}_{\mu}\right)$ отражает интерференцию

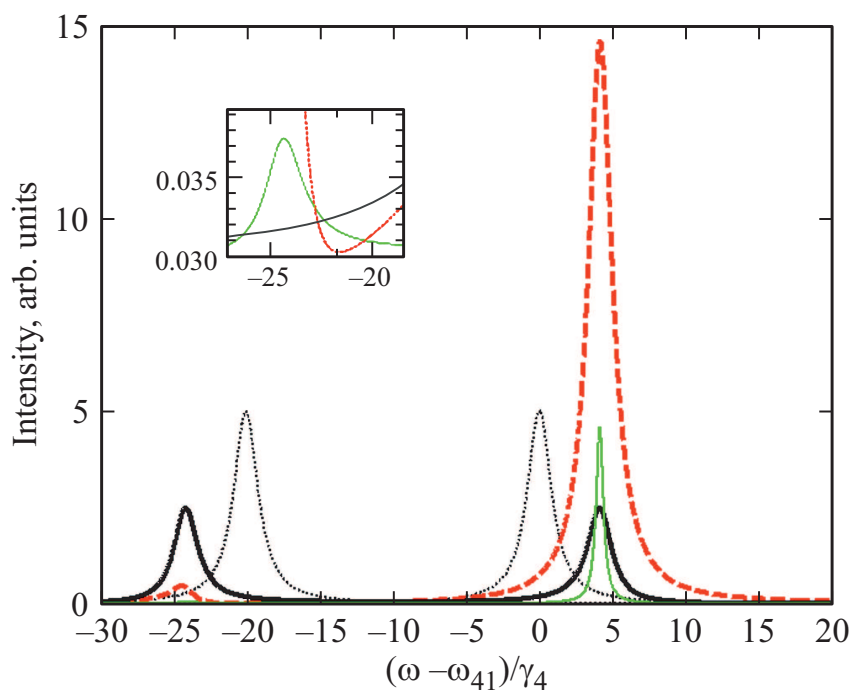

Рис. 2. Пример спектров флуоресценции свободных частиц и спектров неселективной и селективной флуоресценции пары взаимодействующих частиц. Тонкая сплошная кривая изображает уменьшенный в 5 раз контур $S_{12 \mu}$, толстые линии увеличенные в 20 раз контуры $S_{12 \sigma}$ (штриховая линия) и $S_{12 v}$ (сплошная линия). Пунктирными кривыми показаны увеличенные в 5 раз $S_{A}\left(\omega=\omega_{\sigma}\right)$ и $S_{B}\left(\omega=\omega_{\nu}\right)$. Во вставке увеличенный фрагмент графиков.

$\mu$-флуоресценции, обусловленной излучением фотона $\mu$ как частицей $A$, так и частицей $B$ при переходе частиц в коллективное основное состояние $|12\rangle$.

Спектр $S_{12 \mu}\left(\bar{\omega}_{\sigma}\right)$ имеет вид:

$$
\begin{aligned}
\left|b_{12 \sigma}\left(\bar{\omega}_{\sigma}, \infty\right)\right|^{2} \equiv & S_{12 \sigma}\left(\bar{\omega}_{\sigma}\right) \\
= & \left|V_{1 \sigma}^{40}\right|^{2}\left[\left(\bar{\omega}_{\sigma}+2 \tilde{\Delta}\right)^{2}+\gamma_{3}^{2}\right] /\left|F_{\sigma}\left(\bar{\omega}_{\sigma}\right)\right|^{2} \\
\left|F_{\sigma}\left(\bar{\omega}_{\sigma}\right)\right|^{2}= & \hbar^{2}\left[\left(\bar{\omega}_{\sigma}+\tilde{\Delta}-\delta\right)^{2}+(\bar{\gamma}+\eta)^{2}\right] \\
& \times\left[\left(\bar{\omega}_{\sigma}+\tilde{\Delta}+\delta\right)^{2}+(\bar{\gamma}-\eta)^{2}\right] .
\end{aligned}
$$

Так же как и спектр неселективной флуоресценции $S_{12 \mu}\left(\bar{\omega}_{\mu}\right)$, спектр $S_{12 \sigma}\left(\bar{\omega}_{\sigma}\right)$ состоит из двух компонент, обусловленных расщеплением линии $\omega_{41}$. Контуры этих компонент имеют максимумы при $\bar{\omega}_{\sigma}=\delta-\tilde{\Delta}$ и $\bar{\omega}_{\sigma}=-\delta-\tilde{\Delta}$, а расстояние между этими максимумами $\cong 2 \delta>2 \tilde{\Delta}$. Обозначим эти контуры как $S_{A I \sigma}\left(\bar{\omega}_{\sigma}\right)$ и $S_{A I I \sigma}\left(\bar{\omega}_{\sigma}\right)$. Контур $S_{A I \sigma}\left(\bar{\omega}_{\sigma}\right)$ сдвинут относительно контура $S_{A}\left(\bar{\omega}_{\sigma}\right)$ линии собственной флуоресценции изолированной частицы $A$ в сторону большей частоты, а контур $S_{A I I \sigma}\left(\bar{\omega}_{\sigma}\right)$ сдвинут в сторону меньшей частоты настолько, что его максимум располагается при меньшем значении частоты, чем частота максимума контура собственной флуоресценции частицы $B$.

Спектр $S_{12 v}\left(\bar{\omega}_{v}\right)$ имеет вид:

$$
\left|b_{12 v}\left(\bar{\omega}_{v}, \infty\right)\right|^{2} \equiv S_{12 v}\left(\bar{\omega}_{v}\right)=\left|V_{2 v}^{30}\right|^{2} w^{2} /\left|F_{v}\left(\bar{\omega}_{v}\right)\right|^{2},
$$

где вследствие отмеченного раньше равенства $F_{\sigma}\left(\bar{\omega}_{\sigma}\right)=F_{\nu}\left(\bar{\omega}_{\nu}\right)$ зависимость $\left|F_{\nu}\left(\bar{\omega}_{\nu}\right)\right|^{2}$ имеет такой же вид, как приведенная выше зависимость $\left|F_{\sigma}\left(\bar{\omega}_{\sigma}\right)\right|^{2}$. 
Спектр $S_{12 v}\left(\bar{\omega}_{v}\right)$ состоит из двух компонент, обусловленных расщеплением линии $\omega_{32}$ спонтанной собственной флуоресценции линии изолированной частицы $B$ под влиянием ее взаимодействия с частицей $A$. Обозначим эти компоненты $S_{B I v}\left(\bar{\omega}_{v}\right)$ и $S_{B I I v}\left(\bar{\omega}_{\nu}\right)$. Как отмечено раньше, зависимость $\left|F_{\nu}\left(\bar{\omega}_{\nu}\right)\right|^{2}$ имеет такой же вид, как приведенная выше зависимость $\left|F_{\sigma}\left(\bar{\omega}_{\sigma}\right)\right|^{2}$, так что контур $S_{B I v}\left(\bar{\omega}_{\nu}\right)$ перекрывается с контуром $S_{A I \sigma}\left(\bar{\omega}_{\sigma}\right)$, а контур $S_{B I I v}\left(\bar{\omega}_{\nu}\right)-$ с контуром $S_{A I I \sigma}\left(\bar{\omega}_{\sigma}\right)$, при том, что максимальные значения этих перекрывающихся контуров достигаются при одинаковой частоте, их полуширина и значения в максимумах, вообще говоря, существенно различаются, так же как и относительные значения максимумов контуров $S_{B I \nu}\left(\bar{\omega}_{v}\right)$ и $S_{B I I v}\left(\bar{\omega}_{\nu}\right)$ по сравнению с относительными значениями максимумов контуров $S_{A I \sigma}\left(\bar{\omega}_{\sigma}\right)$ и $S_{A I I \sigma}\left(\bar{\omega}_{\sigma}\right)$.

Иллюстрация отмеченных особенностей вида контуров спектральных линий флуоресценции при конкретных значениях параметров частиц см. на рисунках в следующей части статьи.

Вследствие различия интенсивности контуров $S_{12 \sigma}\left(\bar{\omega}_{\sigma}\right)$ и $S_{12 v}\left(\bar{\omega}_{v}\right)$ отличаются их интегральные значения. Так, выполняя интегрирование по телесному углу вокруг направления распространения фотона $\sigma$ c учетом двух состояний его поляризации, для интегрального спектра полной $\sigma$-флуоресценции имеем

$$
\int_{-\infty}^{+\infty} S_{12 \sigma}\left(\bar{\omega}_{\sigma}\right) d \omega_{\sigma}=\frac{2\left(\bar{\gamma}^{2}-\eta^{2}\right)\left(\delta^{2}+\bar{\gamma}^{2}\right)-\bar{\gamma} \gamma_{3} w^{2}}{2\left(\bar{\gamma}^{2}-\eta^{2}\right)\left(\delta^{2}+\bar{\gamma}^{2}\right)}
$$

а для интегрального спектра полной $v$-флуоресценции имеем

$$
\int_{-\infty}^{+\infty} S_{12 v}\left(\bar{\omega}_{v}\right) d \omega_{v}=\frac{\bar{\gamma} \gamma_{3} w^{2}}{2\left(\bar{\gamma}^{2}-\eta^{2}\right)\left(\delta^{2}+\bar{\gamma}^{2}\right)}
$$

Отличие значения интегрального спектра $\sigma$-флуоресценции от значения интегрального спектра $v$-флуоресценции согласуется с отмеченным во Введении представлением о рассмотрении разделения общей вероятности заселенности основного коллективного состояния частиц $|12\rangle$ при регистрации фотона флуоресценции на вероятности заселения конечного состояния той или иной частицы $(|1\rangle$ или $|2\rangle)$. Применяя это представление, приведенную величину интегрального спектра полной $\sigma$-флуоресценции можно рассматривать как отражение заселенности

$$
P_{1} \equiv \int_{-\infty}^{+\infty} S_{12 \sigma}\left(\bar{\omega}_{\sigma}\right) d \omega_{\sigma}
$$

основного состояния частиц $A$, в котором они оказались в результате регистрации фотонов $\sigma$-флуоресценции, сопровождающейся окончанием спектроскопических переходов $4 \rightarrow 1$ в каждой частице $A$ ансамбля рассматриваемых пар частиц. Соответственно величину интегрального спектра полной $v$-флуоресценции можно рассматривать как отражение заселенности

$$
P_{2} \equiv \int_{-\infty}^{+\infty} S_{12 v}\left(\bar{\omega}_{v}\right) d \omega_{v}
$$

основного состояния частиц $B$, т.е. иметь информацию относительно селективного заселения основного состояния частиц в ансамбле рассматриваемых пар частиц, и рассматривать $P_{1}$ и $P_{2}$ как вклады в общую заселенность коллективного основного состояния $|12\rangle$ пары частиц $P_{12}=1$, отражая сохранение нормировки амплитуд $b_{k}(t), k \div 420,130,12 \mu$, и амплитуд $b_{k}(t)$, $k \div 420,130,12 \sigma, 12 v$, полученных при решении приведенных выше систем уравнений Шредингера. Так что можно сказать, что полученные выражения $S_{12 \sigma}\left(\bar{\omega}_{\sigma}\right)$ и $S_{12 v}\left(\bar{\omega}_{v}\right)$ отражают отличие спектра излучения частицей $A$, взаимодействующей с частицей $B$, от спектра излучения частицей $B$, взаимодействующей с частицей $A$, и различие их интенсивностей является проявлением селективности рассматриваемой флуоресценции. Такое различие может быть использовано, например, при изучении выделения флуоресценции от одной из пары квантовых точек, которое проводилось в [14] по анализу результатов многократного измерения мерцания этой флуоресценции для различных расстояний между этими квантовыми точками при регистрации фотонов матричным детектором в составе люминесцентного микроскопа.

Например, для частиц с параметрами $\gamma_{3}=3, \tilde{\Delta}=10$, $w=5$ (в единицах $\left.\gamma_{4}\right)$ имеем $\delta=11.17, \eta=-0.89$, $P_{1}=0.60$ и $P_{2}=0.40-$ невысокая эффективность вторичного фотопревращения частиц, а для частиц с параметрами $\gamma_{3}=3, \tilde{\Delta}=10, w=20$ имеем $\delta=22.34$, $\eta=-0.45, P_{1}=0.11$ и $P_{2}=0.89-$ высокая эффективность вторичного фотопревращения частиц.

\section{Иллюстрация вида контуров спектральных линий}

Приведем рисунки, иллюстрирующие характерные виды полученных контуров спектральных линий флуоресценции на примерах контуров флуоресценции частиц с конкретными значениями констант $\gamma_{3}, \gamma_{(34)}, w, \tilde{\Delta},\left|{ }_{A} V_{1 \sigma}^{40}\right|^{2}$, $\left|{ }_{B} V_{2 v}^{30}\right|^{2}$ которые, так же как значения частоты $\bar{\omega}_{\mu}, \bar{\omega}_{\sigma}$, $\bar{\omega}_{\nu}$, будут взяты в единицах $\gamma_{4}$. Для сравнения приведем также контуры спектральных линий собственной флуоресценции свободных частиц.

Для спектра полной спонтанной флуоресценции свободной частицы $A$ после интегрирования выражения $S_{12 \sigma}\left(\bar{\omega}_{\sigma}, w=0\right)$ по телесному углу вокруг направления 
распространения фотона $\sigma$ (с учетом двух состояний поляризации) имеем следующее выражение:

$$
S_{A}\left(\bar{\omega}_{\sigma}\right)=\gamma_{4}^{2} /\left(\bar{\omega}_{\sigma}^{2}+\gamma_{4}^{2}\right) .
$$

Для спектра полной спонтанной флуоресценции свободной частицы $B$ будем использовать выражение

$$
S_{B}\left(\bar{\omega}_{\nu}\right)=\frac{\gamma_{3}^{2}}{\left(\bar{\omega}_{\nu}+2 \tilde{\Delta}\right)^{2}+\gamma_{3}^{2}}
$$

или

$$
S_{B}\left(\omega_{v}\right)=\frac{\gamma_{3}^{2}}{\left(\omega_{v}-\omega_{32}\right)^{2}+\gamma_{3}^{2}} .
$$

На приведенных ниже рисунках по оси ординат отложены безразмерные значения $S_{k}, k \div 12 \mu, 12 \sigma, 12 \nu$, а по оси абсцисс - частота $\omega-\omega_{41}$ в единицах $\gamma_{4}^{-1}$, значение которой, как отмечено выше, совпадает со значением $\bar{\omega}_{\mu}$ для функций $S_{12 \mu}\left(\bar{\omega}_{\mu}\right)$, значением $\bar{\omega}_{\sigma}$ для функций $S_{12 \sigma}\left(\bar{\omega}_{\sigma}\right)$ и со значением $\bar{\omega}_{v}$ для функций $S_{12 v}\left(\bar{\omega}_{v}\right)$.

Для частиц, характеризуемых параметрами $\gamma_{3}=\gamma_{(34)}=1, \quad w=\tilde{\Delta}=10, \quad$ частотные зависимости $S_{12 \sigma}\left(\omega=\omega_{\sigma}\right), S_{12 v}\left(\omega=\omega_{\nu}\right)$ и $S_{12 \mu}\left(\omega=\omega_{\mu}\right)$ в единицах $\left|{ }_{A} V_{1 \sigma}^{40}\right|^{2}$ и $\left|{ }_{B} V_{2 v}^{30}\right|^{2}$ приведены на рис. 2. Тонкая сплошная кривая изображает контур $S_{12 \mu}$, уменьшенный в 5 раз, толстая штриховая - контур $S_{12 \sigma}$, увеличенный в 20 раз, утолщенная сплошная - контур $S_{12 v}$, также увеличенный в 20 раз. Пунктирными кривыми изображены увеличенные в 5 раз $S_{A}\left(\omega=\omega_{\sigma}\right)$ с максимумом при $\omega=\omega_{41}$ и $S_{B}\left(\omega=\omega_{\nu}\right)$ с максимумом при $\omega=\omega_{32}$.

Этот рисунок иллюстрирует проявление общих закономерностей расположения контуров, которые отмечены в предыдущей части статьи. Это прежде всего совпадение частоты максимумов контуров $S_{12 \sigma}$ и $S_{12 v}$. Так, контуры $S_{A I}\left(\bar{\omega}_{\sigma}\right)$ и $S_{B I}\left(\bar{\omega}_{\sigma}\right)$ имеют максимумы при одном и том же значении $\omega-\omega_{41}=4.14$, а контуры, $S_{A I I}\left(\bar{\omega}_{\sigma}\right)$, и $S_{B I I}\left(\bar{\omega}_{\sigma}\right)-$ при $\omega-\omega_{41}=-24.14$, а поскольку для рассматриваемых частиц $\delta=14.14 \cong \alpha$ и $\eta \cong \beta=0$, то и контуры $S_{A I \mu}\left(\bar{\omega}_{\mu}\right), S_{A I I \mu}\left(\bar{\omega}_{\mu}\right)$ имеют максимумы соответственно при тех же значениях частоты (контур $S_{A I I \mu}\left(\bar{\omega}_{\mu}=-24.14\right)$ см. во вставке). Рисунок иллюстрирует также существенное различие относительных значений максимумов контуров. Так, значения максимумов контуров $S_{A I \mu}\left(\bar{\omega}_{\mu}\right)$ и $S_{A I}\left(\bar{\omega}_{\sigma}\right)$ при $\omega-\omega_{41}=4.14$ намного превосходят значения максимумов контуров $S_{A I I \mu}\left(\bar{\omega}_{\mu}\right)$ и $S_{A I I}\left(\bar{\omega}_{\sigma}\right)$ при $\omega-\omega_{41}=-24.14$, в то время как значения максимумов контуров $S_{B I}\left(\bar{\omega}_{\sigma}\right)$ и $S_{B I I}\left(\bar{\omega}_{\sigma}\right)$ одинаковы и при $\omega-\omega_{41}=4.14$, и при $\omega-\omega_{41}=-24.14$.

Рисунок 3 иллюстрирует различие вида контуров линий селективной флуоресценции при невысокой и высокой эффективности вторичного фотопревращения частиц с параметрами, приведенными в конце предыдущей части статьи.

На этом рисунке тонкие кривые изображают контуры линий при невысокой эффективности вторичного фотопревращения частиц, при этом штриховая кривая

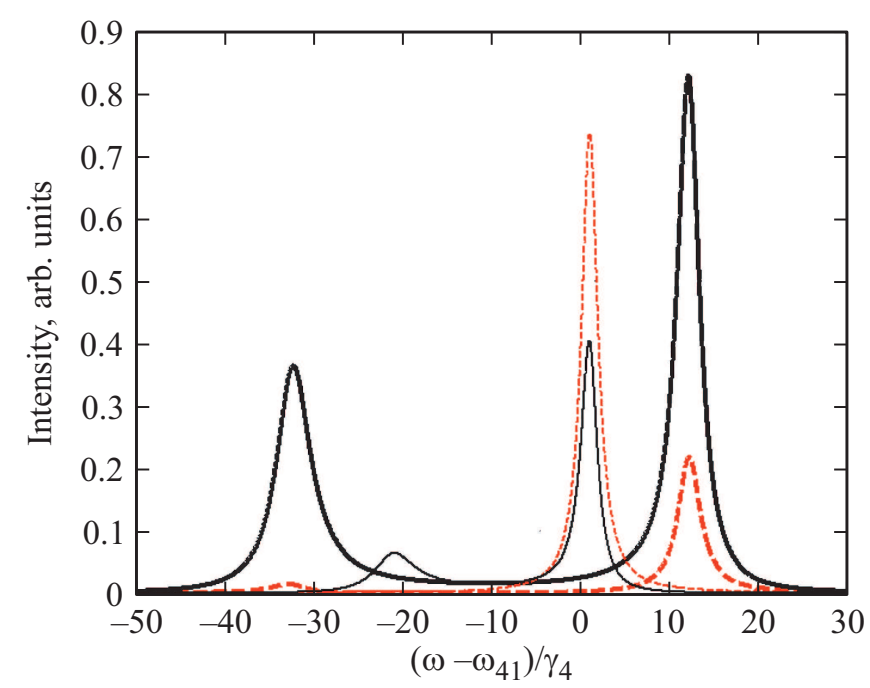

Рис. 3. Пример спектров флуоресценции частиц $S_{12 \sigma}\left(\omega=\omega_{\sigma}\right)$ (штриховые линии) и $S_{12 v}\left(\omega=\omega_{v}\right)$ (сплошные линии, масштаб увеличен в 10 раз) при невысокой (тонкие линии) и высокой (толстые линии) эффективности вторичного фотопревращения частиц.

изображает $S_{12 \sigma}\left(\omega=\omega_{\sigma}\right)$, а сплошная $-S_{12 v}\left(\omega=\omega_{\nu}\right)$, увеличенную в 10 раз. Утолщенные кривые изображают контуры линий при высокой эффективности вторичного фотопревращения частиц, при этом штриховая кривая изображает $S_{12 \sigma}\left(\omega=\omega_{\sigma}\right)$, а сплошная $-S_{12 v}\left(\omega=\omega_{n u}\right)$, также увеличенную в 10 раз, как и $S_{12 v}\left(\omega=\omega_{v}\right)$ при невысокой эффективности.

Сравнение тонких и утолщенных кривых иллюстрирует увеличение расщепления линий и увеличение максимумов соответствующих контуров линий первоначально невозбужденной частицы с ростом константы взаимодействия частиц.

\section{Заключение}

Полученные выражения для спектральных характеристик селективной флуоресценции и результат их анализа могут рассматриваться как вклад в формализм определения параметров взаимодействующих частиц на основе спектрального метода, дополнительного к методу квантовых биений интенсивности флуоресценции пары частиц (применяемому в работе [13]), и к методу, основанному на определении мерцания флуоресценции пары частиц (применяемому в работе [14]).

\section{Конфликт интересов}

Автор заявляет, что у него нет конфликта интересов.

\section{Список литературы}

[1] Georgescu I.M., Ashhab S., Nori F. // Rev. Mod. Phys. 2014. V. 86. P. 154. 
[2] Maiolo T.A.C., Della Sala F., Martina L., Soliani G. // Theor. Math. Phys. 2007. V. 152. P. 1156.

[3] Кемпфер Ф. Основные положения квантовой механики. М.: Мир, 1967. 391 c.; Kaempffer F.A. Concepts in quantum mechanics. New York and London: Academic Press., 1965.

[4] Plenio M.B., Knight P.L. // Rev. Mod. Physics. 1998. V. 70. N 1. P. 101.

[5] Менский М.Б. Квантовые измерения и декогеренция. Модели и феноменология. М.: ФИЗМАТЛИТ, 2001. 232 с.

[6] Скалли М.О., Зубайри М.С. Квантовая оптика. М.: ФИЗМАТЛИТ, 2003. 504 c.; Scully M.O., Zubairy M.S. Quantum optics. Cambridge University Press, 1997.

[7] Pokorny F., Zhang Chi, Higgins G. Cabello A., Kleinmann M., Hennrich M. // Phys. Rev. Lett. 2020. V. 124. P. 080401.

[8] Башаров А.М., Маныкин Е.А. // Опт. и спектр. 2004. Т. 96. B. 1. C. 91; Basharov A.M., Manykin E.A. // Opt. Spectrosc. 2004. V. 96. N 1. P. 81.

[9] Макомбер Джс.Д. Динамика спектроскопических переходов. М.: Мир, 1979. 347 с.; Macomber J.D. The Dynamics of spectroscopic transitions. New York: John Wiley and Sons, 1976.

[10] Нильсен М., Чанг И. Квантовые вычисления и квантовая информация. М.: Мир, 2006. 822 c.; Nielsen M.A., Chuang I.L. Quantum Computation and Quantum Information. Cambridge University Press, 2000.

[11] Monroe C., Campbell W.-C., Duan L.-M., Gong Z.-X., Gorshkov A.V., Hess P.W., Islam R., Kim K., Linke N.M., Pagano G., Richerme P., Senko C. // Rev. Mod. Phys. 2021. V. 93. P. 025001.

[12] Bashkirov E.K. // Intern. J. Theor. Physics. 2019. V. 58. P. 2346.

[13] Мохов А.И., Макаров А.А. // Опт. и спектр. 2019. Т. 127. B. 1 C. 13; Mokhov A.I., Makarov A.A. // Opt. Spectrosc. 2019. V. 127. N 1. P. 7.

[14] Еремчев И.Ю., Лозинг Н.А., Баев А.А., Тарасевич А.О., Гладуш М.Г., Рожсенцов А.А., Наумов А.В. // Письма в ЖЭТФ. 2018. T. 108. В. 1. C. 26; Eremchev I.Yu, Lozing N.A., Baev A.A., Tarasevich A.O., Gladush M.G., Rozhentsov A.A. Naumov A.V. // JETP Lett. 2018. V. 108. P. 30.

[15] Das S., Agarwal G.S. // J. Phys. B: At. Mol. Opt. Phys. 2009. V. 42. P. 141003.

[16] Гайтлер В. Квантовая теория излучения. М.: Изд-во иностр. лит., 1956. 491 с.; Heitler $W$. The quantum theory of the radiation. Oxford at the Clarendon Press, 1954.

[17] Weiss U. Quantum Dissipative Systems. Singapore: World Scientific, 2012. 448 p.

[18] Фейнман $P$., Хибс А. Квантовая механика и интегралы по траекториям. М.: Мир, 1968. 382 c.; Feynman R.P., Hibbs A.R. Quantum mechanics and path integrals. New York: McGraw-Hill Book Company, 1965. 